\title{
The management of $B C G$ failure in non-muscle-invasive bladder cancer: an update
}

\author{
Alexandre R. Zlotta, MD, FRCSC,; Neil E. Fleshner, MD, FRCSC; ${ }_{i}^{* \neq}$ Michael A. Jewett, MD, FRCSC ${ }^{* \neq}$
}

\begin{abstract}
Up to $40 \%$ of patients with non-muscle-invasive bladder cancer (NMIBC) will fail intravesical bacillus Calmette-Guérin (BCG) therapy. There is unfortunately no current gold standard for salvage intravesical therapy after appropriate BCG treatment. Indeed, outcomes are at best suboptimal. The vast majority of low-grade NMIBC are prone to recur but very rarely progress. Failure after intravesical BCG in these patients is usually superficial and lowgrade. At the other end of the spectrum, failure to respond to BCG in high-risk T1 bladder cancer and/or carcinoma in situ (CIS or $\mathrm{TIS}$ ) is more problematic, since those tumours often have the potential to progress to muscle invasion. In these cases, radical cystectomy remains the mainstay after BCG failure. With appropriate selection, certain patients who "fail" BCG (but with favourable risk factors) can be managed with intravesical regimens, including repeated BCG, BCG plus cytokines, intravesical chemotherapy, thermochemotherapy or new immunotherapeutic modalities. In this review, reasons explaining BCG failure, how to define BCG failure, optimal risk stratification and prediction of response and management of BCG failures are discussed.
\end{abstract}

Can Urol Assoc J 2009;3(Supp14):S199-205

\section{Introduction and issues}

The choice of an appropriate course of action following the failure of intravesical bacillus Calmette-Guerin (BCG) treatment is a controversial management issue for both patients with non-muscle-invasive bladder cancer (NMIBC) and their physicians. It represents a frequent dilemma, since approximately $30 \%$ to $40 \%$ of patients do not respond to this therapy. ${ }^{1,2}$ Treatment options should be divided between those patients with low-grade disease who do not respond to treatment and those with high-grade disease and/or progress who do not respond, the latter being associated with poor prognosis. Although grouped under the same terminology, NMIBC consists of 2 biologically different diseases. Most low-grade NMIBC are likely to recur but very rarely progress. Failure after intravesical BCG in these patients is usually superficial and low grade. At the other end of the spectrum, failure to respond to BCG in high-risk T1 bladder cancer and/or carcinoma in situ (CIS or TIS) is more problematic, since these tumours often progress to muscle invasion. ${ }^{3}$

The European Association of Urology (EAU) guidelines on bladder cancer recommend BCG for intermediate-risk patients for whom intravesical chemotherapy is unsuccessful and as the first treatment choice in high-risk patients. ${ }^{3}$ However, the EAU guidelines recommend intravesical chemotherapy in low-risk patients because of an enhanced toxicity profile.

In North America and Canada, many urologists administer BCG as a first-line of defence in low-grade disease. ${ }^{4}$ BCG failure usually carries a risk of recurrence but rarely of progression. For low-grade failure, intravesical chemotherapy is a valid option. ${ }^{1}$ This scenario is different from that where BCG intravesical therapy is unsuccessful in highrisk $\mathrm{T} 1$ disease and/or $\mathrm{CIS}$, where a risk of progression may sometimes reach $50 \%{ }^{1,2}$ Controversy exists over the most appropriate treatment for high-risk superficial bladder cancer $^{1}$ (stage T1, grade G3 or the new classification of high grade). ${ }^{2,3}$ Immediate cystectomy offers the best chance for survival but may be associated with impaired quality of life (QOL) compared with conservative therapy. A second option is administration of BCG. Responders to BCG retain their bladder and are spared cystectomy and its associated complications, thereby maintaining their QOL. When BCG fails, even as new drugs and therapeutic modalities are investigated and experience with a second BCG regimen and combination immunotherapy increases, there may be a natural tendency to delay definitive local therapy and therefore expose patients to a greater risk of progression to invasive and potentially metastatic disease. ${ }^{5}$ The concern is that a physician may wait too long to recommend cystectomy. Recent data suggest that when cystectomy is performed following conservative attempts in cases of high-risk NMIBC, the 5-year postoperative survival rate is lower than reported in recent studies, especially when cystectomy is performed on a patient with disease that has progressed to $\mathrm{T} 2$, as opposed to patients with T1 NMIBC. ${ }^{5}$

Kulkarni and colleagues recently evaluated 2 treatment strategies for high-risk T1G3 bladder cancer using a decisionanalytic Markov model ${ }^{6}$ as follows: (1) Immediate cystectomy with neobladder creation versus (2) conservative management with intravesical BCG and delayed cystectomy in 
individuals with resistant or progressive disease. The authors demonstrated that younger patients with high-risk T1G3 bladder had a greater life expectancy and QOL following immediate cystectomy. ${ }^{6}$ The decision to pursue immediate cystectomy versus conservative therapy could be based on discussions that consider patient age, comorbidity and individual preference. Patients over the age of 70 or those who place high value on bladder preservation may benefit from a more conservative initial therapeutic approach.

Conversely, in a large series of primary high-grade T1 patients from Toronto and Rotterdam $(n=136)$ that were managed conservatively and followed for more than 5 years (median 6.5 years), progression was observed in 30\% of cases but the remaining $70 \%$ did not progress. ${ }^{7}$ These observations highlight some of the dilemmas surrounding the management of high-risk NMIBC and BCG failure.

\section{Reasons for BCG failure}

There are various factors that may explain BCG failure. Although it is not the scope of this review, these may include:

Insufficient or excess BCG. BCG is an immunotherapy in which a $\mathrm{TH} 1$-type response is usually driven both locally in the bladder and in the peripheral circulation, with the production of IL-2, IFN- $\gamma$, IL-12 and IL-18 among other cytokines. ${ }^{8}$ The net effect of chemokine signals is an escalating recruitment of monocytic and granulocytic leukocytes into the bladder with each successive weekly BCG instillation. When an important and effective TH1 cellular response has been triggered (which plays a key role), cellular activation leads to amplification of effector cells capable of eliminating bladder tumour cells and producing cytokines to regulate immune response. Several lines of evidence suggest that insufficient BCG as well as excess quantities of BCG may impair BCG efficacy. Low BCG doses lead to an almost exclusively cell-mediated Th1 response. In contrast, higher doses induce a mixed $\mathrm{TH} 1 / \mathrm{TH} 2$ response. $^{9}$ Similar to what is observed after BCG vaccination, waning immunity might not be due to induction of insufficient $\mathrm{TH} 1$ immune activity, which is necessary for BCG activity, but rather to the presence of other mechanisms that undermine the efficacy of the $\mathrm{TH} 1$ response, rather than other inappropriate $\mathrm{TH} 2$ responses when large quantities of BCG are administered. Previous exposure to mycobacteria (specifically, environmental bacteria) may play a role. Assessment of the immune reactivity against mycobacterial antigens (BCG is a mycobacteria)-which is rarely if ever assessed in patients with NMIBC - may also be of significance in determining an appropriate BCG dose. ${ }^{10}$ Although it is wellestablished that some patients fail to respond because they did not receive enough BCG (often because of tolerability issues), it is also likely that some patients receive too much BCG. Recently, low doses (sometimes one-tenth or even one-thirtieth of the dose) have been proposed for maintenance therapy.

\section{Premature evaluation}

Occult invasive or metastatic disease. Micrometastatic disease diagnosis is notoriously suboptimal. Patients with NMIBC may already harbour micrometastatic disease. The longterm outcome of patients initially receiving cystectomy at the T1 stage suggests that the prevalence of micrometastatic disease might often be underappreciated..$^{11}$

\section{Failure of $B C G$ contact with the target}

Gradual waning of the immune response. Intravesical BCG instillations induce a transient (less than 6 months) peripheral immune activation against BCG antigens. Reactivation is observed in most cases after additional BCG courses. The absence of long-lasting immune activation after a single 6-week course of BCG could be related to the increased clinical efficacy observed with BCG maintenance instillations. ${ }^{12,13,14}$

Inadequate immune response. There is strong evidence that the success of BCG therapy might be due to a preferential induction of a $\mathrm{TH}-1$ response (detected in the urine by analysis of relevant cytokines). ${ }^{15,16}$ Although somewhat controversial, $\mathrm{TH}-2$ responses detected either in the peripheral circulation or locally in the bladder are associated with poorer outcomes and might explain failure to respond to BCG therapy. ${ }^{17}$

Natural resistance-associated macrophage protein (NRAMP1) gene polymorphisms. The NRAMP1 gene has been implicated in susceptibility to infectious diseases and in response to BCG. Data suggest implication of the NRAMP1 gene in bladder cancer recurrence and response to BCG. ${ }^{18}$

Unresponsive tumour. Many recent investigations have determined whether biological markers might predict disease progression and/or response to treatment. ${ }^{19}$ An excellent review was provided a couple of years ago by Saint and colleagues. ${ }^{20}$

\section{Defining BCG failure}

When should a case be labelled BCG refractory? BCG failure is inconsistently defined in the literature. There is no clear answer to the frequent clinical dilemma of choosing when to abandon BCG in favour of another strategy. Herr suggested that a total treatment and follow-up time of at least 6 months is necessary to identify early BCG failure. $\mathrm{He}$ also suggested that switching to an alternative regimen before 6 months is probably premature, may ignore the delayed therapeutic effects of repeat transurethral resection combined with BCG, and artificially inflate response rates of salvage regimens. ${ }^{21}$ However, the poor prognosis 
of an early high-grade $\mathrm{T} 1$ recurrence at the first post-BCG 3 -month cystoscopy in high-risk NMIBC may temper this view when it is associated with CIS.

A simple definition of BCG failure would be tumour recurrence at 3 months or tumour progression at any time, but many questions remain. What about a patient with marked reduction in grade stage and number of tumours who subsequently presents with a low-grade recurrence?

The Canadian Guidelines for Treatment of Non-Muscle Invasive Bladder Cancer define BCG failure as: 1) the presence of high-grade NMIBC at 6 months from the time of a transurethral resection of a bladder tumour (TURBT); at 3 months if the initial tumour is T1G3/T1HG; or worsening of the disease (higher grade, stage or number of recurrences, appearance of $\mathrm{CIS}$ ) while on BCG therapy despite initial response to BCG (level 2 evidence). ${ }^{22} \mathrm{~A}$ second induction course may achieve a $30 \%$ to $50 \%$ response rate. ${ }^{23,24}$ A more uniform reporting mechanism to improve the definition of BCG failure in patients has been proposed as follows: ${ }^{25}$ 1) BCG-refractory disease when there is failure to achieve a disease-free state at 6 months following initial BCG therapy with either maintenance or retreatment at 3 months because of persistent or rapidly recurrent tumour; 2) BCG-resistant disease when there is recurrence or persistence at 3 months following an induction cycle; 3) BCGrelapsing disease when the disease recurs after the patient is disease-free for 6 months; and 4) BCG-intolerant disease when the disease recurs following administration of a less than adequate course of therapy because of a serious adverse event or symptomatic intolerance that requires discontinuation of further BCG therapy.

Indeed, patients may not tolerate BCG (BCG intolerance) because of its side effects. When this occurs during the first 6 instillations, it is not precisely a BCG failure; ${ }^{1}$ rather, it is because BCG therapy was insufficient. Although intravesical BCG has more severe and more frequent side effects compared to intravesical chemotherapy, the number of patients that discontinue BCG instillations during the induction course is significantly different between Europe and North America, at least as reported in the South West Oncology Group (SWOG) trial. In the European Organization for Research and Treatment of Cancer (EORTC) study in which 487 patients received 36 months of BCG, ${ }^{26}$ only $20 \%$ of patients discontinued BCG due to local and/or systemic side effects. Local toxicity remained constant during maintenance therapy. Fewer than $5 \%$ of patients did not complete the induction course, indicating that they never received sufficient BCG therapy. In the North American SWOG trial, ${ }^{27}$ in sharp contrast with results of the EORTC study, only $27 \%$ of patients completed the 3-year maintenance course. The immune status of the patients and possibly their immune reactivity against mycobacterial antigens might explain the differ- ences between the experience reported with BCG maintenance in Europe and North America.

Patients with CIS are considered BCG-refractory when biopsies and cytology do not normalize after 1 initial course of 6 weekly instillations and following at least 1 second course after 3 months, because a second course of BCG can turn a positive cytology into a negative cytology in an additional $10 \%$ to $20 \%$ of cases despite an absence of response after the first 6 months. In a recent meta-analysis of patients with CIS, results following the administration of BCG indicated that $68.1 \%$ had a complete response and $46.7 \%$ remained disease-free after a median follow-up of 3.6 years. ${ }^{28}$

\section{Prediction of BCG failure}

What is the best method for predicting BCG failure? With complex interactions between mycobacteria, a host and a tumour, it is unlikely that one single parameter could be predictive for all patients, regardless of their immunological and tumour background. ${ }^{29}$ Although host, tumour and immunologic parameters can be useful, no single prognostic factor is capable of predicting a positive response. An excellent review by F. Saint ${ }^{30}$ adequately summarizes the knowledge on prognostic parameters of remission versus relapse following BCG therapy.

Clinical risk factors are the easiest to assess by urologists. The recent EORTC tables are useful for predicting the risk of recurrence and progression of NMIBC according to various parameters such as number of tumours, prior recurrence rates, T category and presence of CIS and grade. ${ }^{31}$ Of significance, these tables were developed based on the outcomes of several large EORTC studies, which not only excluded many BCG arms but also were conducted prior to the BCG maintenance era and before recommendations to perform a second TURBT in high-risk patients. Nevertheless, they are of great practical value and are physician-friendly. Another important clinical prognostic factor is the tumour status 3 months following initial TURBT. ${ }^{32}$ Complementing the results observed in the EORTC studies by analyzing studies that only administered BCG, the Spanish Club Urológico Español De Tratamiento Oncológico (CUETO) group evaluated the prognostic factors of recurrence and progression following intravesical adjuvant BCG in 1062 patients with NMIBC from 4 randomized Phase III studies. ${ }^{32}$ Most patients received BCG once weekly for 6 consecutive weeks and short-term BCG maintenance (once every 2 weeks for 6 weeks). Significant independent predictors for recurrence were female gender, history of recurrence, multiplicity and presence of associated CIS. Age, history of recurrence, high grade, T1 stage and recurrence at first cystoscopy were independent predictors of progression.

Substaging in T1 NMIBC may also be predictive of BCG failure. ${ }^{33}$ Van Rhijn, Van der Kwast and colleagues combined 
patients from Toronto and Rotterdam with primary highgrade NMIBC patients treated with BCG and found that substaging was a very significant predictor of BCG recurrence and progression when separating minimal and extensive pT1 high-grade NMIBC (manuscript in preparation).

\section{Conservative treatment options in patients with BCG failure}

\section{Chemotherapy following BCG failure}

Although intravesical chemotherapy is a valid option for low-grade NMIBC BCG failure, the efficacy of chemotherapeutic agents is far from established in high-risk NMIBC. In a Scandinavian study, only 4 of 21 patients with BCG failure that were switched to MMC therapy remained recurrence-free. ${ }^{34}$

Recently, gemcitabine, which is considered standard treatment in systemic therapy for advanced bladder cancer, has been evaluated in the management of superficial disease. Dalbagni and colleagues reported on a Phase I-II trial of gemcitabine in patients refractory to BCG. Patients were considered at high risk for progression and were not candidates for cystectomy. Of 18 patients, 7 had a complete response (defined as negative cytology post-therapy), whereas 4 patients exhibited partial responses (defined as negative biopsies following therapy but with persistent positive cytology). ${ }^{35}$ We have had limited positive experience with intravesical gemcitabine in patients with very aggressive NMIBC following Chinese-herb nephropathy. Patients underwent kidney transplantation and could not receive BCG because of immunosuppression (unpublished data) and therefore received gemcitabine. ${ }^{36}$ In another recent Phase II study using 2000 mg gemcitabine in BCG-refractory patients, 18 of 24 intermediate-risk patients and 7 of 16 high-risk patients remained recurrence-free, confirming the potential benefits of gemcitabine in these patients. ${ }^{37}$

A significantly high marker lesion response of $67.4 \%$ was noted in a recent Phase II study following the administration of 6 intravesical instillations of $4 \mathrm{mg}$ apaziquone (EO9, a synthetic bioreductive alkylating indoloquinone, EOquin). An ongoing study is underway in BCG refractory patients. ${ }^{1}$

Docetaxel has been recently studied as an alternative for BCG-refractory patients unable or unwilling to undergo cystectomy. A retrospective analysis was conducted on 33 patients with refractory NMIBC who received salvage intravesical docetaxel therapy at a single institution. Twenty of $33(61 \%)$ patients had a complete response (CR) after 6 weekly induction treatments. Ten patients with CR were given maintenance docetaxel therapy and 1 patient received maintenance BCG combined with interferon. With a median follow-up of 29 months, 1- and 2-year recurrence-free survival rates were $45 \%$ and $32 \%$, respectively. Toxicity profile was adequate. ${ }^{38}$

In summary, intravesical chemotherapy using new compounds following BCG failure shows some promise but remains highly investigational at this stage.

\section{Device-assisted chemotherapy instillations following BCG failure}

Examples of approaches that improve the efficacy of chemotherapy include a combination of intravesical mitomycin $\mathrm{C}(\mathrm{MMC})$ and an existing gradient between the drug and the bladder wall (electromotive drug administration [EMDA]), and a combination of intravesical MMC and bladder wall hyperthermia (Synergo).

A study of 108 high-risk patients compared EMDA with MMC, classical MMC instillations and BCG alone. ${ }^{39}$ All groups were treated with 1 or 2 six-week courses. The 6-month CR rates were 58\% for MMC with EMDA, 31\% for MMC and $64 \%$ for BCG. No data for the use of MMC with EMDA in BCG failure have been reported to date. Sequential BCG and electromotive mitomycin versus BCG alone for high-risk superficial bladder cancer have also been compared in a randomized controlled trial. ${ }^{40}$

The combination of intravesical hyperthermia and MMC utilizes heating of the bladder wall to a temperature of $42{ }^{\circ} \mathrm{C}$ with a cooled solution of MMC. Heating is performed with special microwave equipment and a special catheter with thermocouples to control for temperature changes. Thermochemotherapy has been reported successful in BCG failures. ${ }^{41}$ In a group of 90 intermediate- and high-risk patients, the 1 - and 2-yr recurrence rates after 1 year of thermochemotherapy were $14.3 \%$ and $24.6 \%$, respectively. No disease progression was observed. In 41 patients failing BCG treatment, the 1- and 2-year recurrence rates were $23 \%$ and $41 \%$. More recent data include a study of 51 patients with CIS treated between 1997 and 2005 in 15 European centres. Thirty-four patients were pretreated with BCG. The initial CR rate was $92 \%$ with a decrease to $50 \%$ after 2 years. ${ }^{42}$ Long-term follow-up trials and additional results are required to ascertain the value of thermochemotherapy in patients with BCG failure.

Finally, photodynamic therapy (PDT) combines photosensitizers that selectively bind to tumours with a powerful intravesical light source to destroy tumours. PDT after oral administration of 5-aminolevulinic acid (5-ALA) has been studied in 24 high-risk BCG-failing patients, including those with CIS. ${ }^{43}$ After an average follow-up of 2 years, 16 patients were free of tumour recurrence, including 4 of 10 BCGfailing patients. 
Immunotherapy and new conservative treatment avenues following BCG failure

We previously demonstrated that many elements of BCG were immunologically active and that there is interest in using its mycobacterial subcomponents rather than the living BCG. ${ }^{44}$ Morales and colleagues, well-known for being the first to report on intravesical BCG use in 1976, assessed the clinical efficacy and safety of another immunomodulator, mycobacterial cell wall-DNA complex (MCC) following intravesical administration in patients with $\mathrm{CIS}^{45}$ Mycobacterial cell wall-DNA complex is a cell wall DNA compound prepared from a culture of the bacterium Mycobacterium phlei. Fifty-five patients received $4 \mathrm{mg}$ or $8 \mathrm{mg}$ MCC emulsion. Patients were previously treated with BCG except for 8 who were treatment-naïve and 2 who received chemotherapy. The CR rate was $27.3 \%$ at weeks 12 , and $26 \%$ in the $4 \mathrm{mg}$ group, while $46.4 \%$ of patients receiving $8 \mathrm{mg}$ had a CR at both time points. Urocidin, a formulation of MCC commercialized by the Canadian company Bioniche, is currently being tested in a Phase III clinical trial in patients with NMIBC with BCG failure and has included the University Health Network/University of Toronto in the trial. It completed recruitment for the initial Phase III registration trial in March 2009.

Interferon- $\alpha$ (IFN- $\alpha$ ) is the most widely studied cytokine. The combination of IFN- $\alpha$ and BCG for BCG failure has been the subject of a large multicentre Phase II trial. ${ }^{46} 1231$ patients with BCG failure were treated with a 6-week induction course of low-dose BCG plus 50 million units of IFN- $\alpha$, followed by 3 additional treatments at 3, 9 and 15 months postinduction. With a median follow-up of 2 years, $48 \%$ remained tumour-free compared to $60 \%$ in the BCG-naïve group treated with standard doses of BCG.

Recent conservative treatment modalities for BCG failures include Vicinium. Vicinium is a fusion protein comprising a humanized scFv specific to EpCAM (epithelial cell adhesion molecule) and a truncated fragment of Pseudomonas exotoxin A. Vicinium specifically targets and induces apoptosis in EpCAM-positive tumours. Data from 46 BCG-refractory or -intolerant patients with CIS of the bladder have been released. ${ }^{47}$ The first 23 patients received Vicinium weekly for 6 weeks. At 3 months, patients with disease $<\mathrm{T} 2$ received a repeat induction course, whereas patients free of disease received maintenance doses weekly for 3 weeks at 3-month intervals. Efficacy data showed CR in 9 of 22 patients at 3 months for the first cohort that received the 6 -week induction regimen. Complete response was maintained in 6 of 19 patients at 6 months and in 3 of 19 patients at 12 months. Complete response was observed in 9 of 23 patients at 3 months for the 12-week induction regimen.

\section{Surgery after BCG failure}

The EAU guidelines recommend cystectomy as the treatment of choice for CIS failing adequate BCG and as an option in other high-risk tumours. ${ }^{3}$ Similarly, the Canadian guidelines state that in patients with high-risk NMIBC with BCG failure, the option of radical cystectomy should be recommended and discussed with the patient (Grade B recommendation). ${ }^{22}$ The guidelines also suggest that immediate cystectomy may be initially offered to patients with T1G3/T1 HG and to patients with high-grade tumours with concomitant $\mathrm{CIS}$ or multiple recurrent high-grade tumours (Grade $\mathrm{C}$ recommendation). The advantage of cystectomy in superficial tumours that failed BCG treatment is obvious. Tumour-specific survival is between $80 \%$ and $90 \%$ at 5 years, and thereby approaches the 5-year tumour-specific survival of patients with superficial bladder cancer. ${ }^{11}$

However, cystectomy for high-risk superficial disease is not only an invasive procedure linked to significant morbidity, but also has a number of other problems associated with it. When patients with NMIBC have a recurrence with invasive disease, a window of opportunity may be overlooked. For instance, in 62 patients with a high-grade recurrence and treated with cystectomy who had failed adequate BCG treatment, the 5-year disease-specific survival rate of progressive patients was only $38 \%$, significantly lower than in those patients without invasive tumour. The authors identified the presence of a tumour in the prostatic urethra before cystectomy as a factor associated with shorter survival. ${ }^{48}$

Preemptive radical cystectomy performed for recurrent T1 disease following intravesical BCG therapy may be associated with improved disease-specific survival and should not be delayed by conservative approaches. ${ }^{49}$ Delay in cystectomy has been shown to result in a poor prognosis. ${ }^{50}$

\section{Conclusion}

Patients with BCG failures are a heterogeneous group and outcomes may be significantly different when low-risk or intermediate-to-high risk NMIBC is considered. In BCGintolerant patients, especially those who never completed an induction course, intravesical therapy combined with another drug at time of recurrence could be beneficial. BCG failure cannot be accurately predicted on an individual basis. However, with clinical and histologic parameters, risk groups can and should be identified because the window of opportunity in patients with BCG failure is narrow; in the case of tumour progression to muscle-invasive cancer, survival rates are suboptimal and should be improved.

Intravesical chemotherapy following BCG failure holds some promise but remains highly investigational. Secondline immunotherapy, such as the combination of BCG and IFN- $\alpha$, is an effective regimen but results should be confirmed. 
Device-assisted intravesical strategies such as PDT, and especially the combination of intravesical hyperthermia and chemotherapy, are candidates to consider in the near future. New compounds are constantly being investigated. Finally, cystectomy results in the best disease-specific survival in patients with BCG failure and should be initially offered to high-risk NMIBC patients.

Further research is essential for discovering new treatments that will improve the outcomes of patients with BCG failure.

From the ${ }^{*}$ Department of Surgery, Division of Urology, University of Toronto; 'Department of Surgery (Urology), Mount Sinai Hospital; ‡Department of Surgical Oncology, University Health Network, Toronto, ON

Competing interests: Dr. Jewett has been on ongoing paid consultant for Viventia Biotech and has received research funds from Bioniche. The other authors have no competing interests to declare.

This paper has been peer-reviewed.

\section{References}

1. Wities JA. Management of BCG failures in superficial bladder cancer: a review. Eur Urol 2006;49:790-7.

2. Barmoshe S, Zlotta AR. Prognosis of TIG3 tumors: clinical factors. Eur Urol Suppl 2004:3:73-8.

3. Babjuk M, Oosterlinck W, Sylvester R, et al. European Association of Urology guidelines on non-muscleinvasive urothelial carcinoma of the bladder. Eur Urol 2008;54:303-14.

4. Joudi FN, Smith BJ, O'Donnell MA, et al. Contemporary management of superficial bladder cancer in the United States: a pattern of care analysis. Urology 2003;62:1083-8.

5. Lerner SP, Tangen CM, Sucharew $H$, et al. Patterns of recurrence and outcomes following induction bacillus Calmette-Guerin for high risk Ta, TI bladder cancer. J Urol 2007;177:1727-31.

6. Kulkarni GS, Finelli A, Fleshner NE, et al. Optimal management of high-risk TIG3 bladder cancer: a decision analysis. PLoS Med 2007;4:e284.

7. Kakiashvili D, Van Rhijn B, Azuero J, et al. Long-term follow-up for primary high grade Tl bladder cancer. Does BCG really prevent progression? Eur Urol Suppl 2009;8:A645.

8. Suttmann H, Jacobsen M, Reiss K, et al. Mechanisms of bacillus Calmette-Guerin mediated natural killer cell activation. J Urol 2004;172(4P+1):1490-5.

9. Power CA, Wei G, Bretscher PA. Mycobacterial dose defines the Th1/Th2 nature of the immune response independently of whether immunization is administered by the intravenous, subcutaneous, or intradermal route. Infect Immun 1998;66:5743-50.

10. Zlotta AR, van Vooren JP, Huygen $K$, et al. What is the optimal regimen for BCG intravesical therapy? Are six weekly instillations necessarry? Eur Urol 2000;37:470-7.

11. Patard JJ, Rodriguez A, Leray E, et al. Intravesical Bacillus Calmette-Guerin treatment improves patient survival in TIG3 bladder tumours. Eur Urol 2002;41:635-41.

12. Decobert $M$, LaRue $H$, Harel $F$, et al. Maintenance bacillus Calmette-Guérin in high-risk nonmuscleinvasive bladder cancer: how much is enough? Cancer 2008;113:710-6.

13. Zlotta AR, Drowart A, Van Vooren JP, et al. Evolution and clinical significance of the T cell proliferative and cytokine response directed against the fibronectin binding antigen 85 complex of bacillus CalmetteGuerin during intravesical treatment of superficial bladder cancer. J Urol 1997;157:492-8.

14. Taniguchi $\mathrm{K}$, Koga $S$, Nishikido $M$, et al. Systemic immune response after intravesical instillation of bacille Calmette-Guérin (BCG) for superficial bladder cancer. Clin Exp Immunol 1999; 115:131-5.

15. de Reijke TM, de Boer EC, Kurth KH, et al. Urinary cytokines during intravesical bacillus Calmette-Guerin therapy for superficial bladder cancer: processing, stability and prognostic value. J Urol 1996;155:477-82.

16. Alexandroff $A B$, Jackson $A M, O^{\prime}$ Donnell $M A$, et al. $B C G$ immunotherapy of bladder cancer: 20 years on. Lancet 1999:353:1689-94.

17. Zlotta AR, Drowart A, Huygen $K$, et al. Humoral response against heat shock proteins and other mycobacterial antigens after intravesical treatment with bacille Calmette-Guérin (BCG) in patients with superficial bladder cancer. Clin Exp Immunol 1997;109:157-65.
18. Decobert $M$, Larue $H$, Bergeron $A$, et al. Polymorphisms of the human NRAMP1 gene are associated with response to bacillus Calmette-Guerin immunotherapy for superficial bladder cancer. I Urol 2006;175:1506-11.

19. Shariat SF, Zlotta AR, Ashfaq R, et al. Cooperative effect of cell-cycle regulators expression on bladder cancer development and biologic aggressiveness. Mod Pathol 2007;20:445-59.

20. Saint F, Salomon L, Quintela R, et al. Do prognostic parameters of remission versus relapse after Bacillus Calmette-Guérin $(B C G)$ immunotherapy exist? analysis of a quarter century of literature. Eur Urol 2003:43:351-60.

21. Herr HW, Dalbagni G. Defining bacillus Calmette-Guerin refractory superficial bladder tumors. J Urol 2003;169:1706-8

22. Kassouf W, Kamat AM, Zlotta A, et al. Canadian Guidelines for Treatment of Non-Muscle Invasive Bladder Cancer : A focus on intravesical therapy. Canadian Urological Association website. http://www.cua.org/guidelines/NonMuscle\%20Invasive\%20Bladder\%20Cancer.pdf. Accessed November 18, 2009.

23. Bui TT, Schellhammer PF. Additional bacillus Calmette-Guérin therapy for recurrent transitional cell carcinoma after an initial complete response. Urology 1997;49:687-90.

24. Jakse G, Hall R, Bono A, et al. Intravesical BCG in patients with carcinoma in situ of the urinary bladder: long-term results of EORTC GU Group phase II protocol 30861. Eur Urol 2001;40:144-50.

25. Nieder AM, Simon MA, Kim SS, et al. Radical cystectomy after bacillus Calmette-Guérin for high-risk Ta, $\mathrm{Tl}$, and carcinoma in situ: defining the risk of initial bladder preservation. Urology 2006;67:737-41.

26. van der Meijden AP, Sylvester RJ, Oosterlinck W, et al. Maintenance Bacillus Calmette-Guerin for Ta TI bladder tumors is not associated with increased toxicity: results from a European Organisation for Research and Treatment of Cancer Genito-Urinary Group Phase III Trial. Eur Urol 2003;44:429-34.

27. Lamm DL, Blumenstein BA, Crissman JD, et al. Maintenance bacillus Calmette-Guerin immunotherapy for recurrent TA, T1 and carcinoma in situ transitional cell carcinoma of the bladder: a randomized Southwest Oncology Group Study. J Urol 2000;163:1124-9.

28. R.J. Sylvester, A.P.M. van der Meijden, J.A. Wities, et al. Bacillus Calmette-Guerin versus chemotherapy in the intravesical treatment of patients with carcinoma in situ of the bladder: a meta-analysis of the published results of randomized clinical trials. J Urol 2005;174:86-92.

29. Shariat SF, Zlotta AR, Ashfaq R, et al. Cooperative effect of cell-cycle regulators expression on bladder cancer development and biologic aggressiveness. Mod Pathol 2007;20:445-59.

30. Saint F, Salomon $L$, Quintela R, et al. Do prognostic parameters of remission versus relapse after Bacillus Calmette-Guérin (BCG) immunotherapy exist? Analysis of a quarter century of literature. Eur Urol 2003:43:351-60.

31. Sylvester RJ, van der Meiiden AP, Oosterlinck W, et al. Predicting recurrence and progression in individual patients with stage Ta TI bladder cancer using EORTC risk tables: a combined analysis of 2596 patients from 7 EORTC trials. Eur Urol 2006:49:466-77.

32. Fernandez-Gomez J, Solsona $E$, Unda $M$, et al. Prognostic factors in patients with non-muscle-invasive bladder cancer treated with bacillus Calmette-Guérin: multivariate analysis of data from four randomized CUETO trials. Eur Urol 2008;53:992-1001.

33. Orsola A, Trias I, Raventos CX, et al. Initial high-grade $\mathrm{Tl}$ urothelial cell carcinoma: feasibility and prognostic significance of lamina propria invasion microstaging (Tla/b/C) in BCG-treated and BCG-nontreated patients. Eur Urol 2005; 48:231-8.

34. Malmstrom PU, Wijkstrom H, Lundholm C, et al. 5-year followup of a randomized prospective study comparing mitomycin C and bacillus Calmette-Guerin in patients with superficial bladder carcinoma. J Urol 1999;161:1124-7.

35. Dalbagni G, Russo P, Sheinfeld J, et al. Phase I trial of intravesical gemcitabine in bacillus CalmetteGuerin-refractory transitional-cell carcinoma of the bladder. J Clin Oncol 2002;20:3193-8.

36. Lemy A, Wissing KM, Rorive $S$, et al. Late onset of bladder urothelial carcinoma after kidney transplantation for end-stage aristolochic acid nephropathy: a case series with 15-year follow-up. Am I Kidney Dis 2008:51:471-7.

37. Gontero $\mathrm{P}$, Casetta $\mathrm{G}$, Maso $\mathrm{G}$, et al. Phase II study to investigate the ablative efficacy of intravesical administration of gemcitabine in intermediate-risk superficial bladder cancer (SBC). Eur Urol 2004;46:339-43.

38. Barlow $\amalg$, McKiernan JM, Benson MC. The novel use of intravesical docetaxel for the treatment of nonmuscle invasive bladder cancer refractory to BCG therapy: a single institution experience. World I Urol 2009. In press.

39. Di Stasi SM, Giannantoni A, Stephen RL, et al. Intravesical electromotive mitomycin C versus passive transport mitomycin C for high risk superficial bladder cancer: a prospective randomized study. J Urol 2003:170:777-82

40. Di Stasi SM, Giannantoni A, Giurioli A, et al. Sequential BCG and electromotive mitomycin versus BCG alone for high-risk superficial bladder cancer: a randomised controlled trial. Lancet Oncol 2006;7:43-51.

41. Van Der Heijden AG, Kiemeney LA, Gofrit ON, et al. Preliminary European results of local microwave hyperthermia and chemotherapy treatment in intermediate or high risk superficial transitional cell carcinoma of the bladder. Eur Urol 2004:46:65-72. 
42. Wijies AJ, Hendricksen $\mathrm{K}$, Gofrit 0 , et al. Intravesical hyperthermia and mitomycin-C for carcinoma in situ of the urinary bladder: experience of the European Synergo working party. World J Urol 2009. In press.

43. Waidelich H, Stepp R, Baumgartner E, et al. Clinical experience with 5-aminolevulinic acid and photodynamic therapy for refractory superficial bladder cancer. J Urol 2001;165:1904-7.

44. Zlotta AR, Van Vooren JP, Denis 0 , et al. What are the immunologically active components of bacille Calmette-Guérin in therapy of superficial bladder cancer? Int I Cancer 2000;87:844-52.

45. Morales A, Phadke K, Steinhoff $G$. Intravesical mycobacterial cell wall-DNA complex in the treatment of carcinoma in situ of the bladder after standard intravesical therapy has failed. J Urol 2009;181:1040-5.

46. O'Donnell MA, Lilli K, Leopold C. Interim results from a national multicenter phase II trial of combination bacillus Calmette-Guerin plus interferon alfa-2b for superficial bladder cancer. J Urol 2004; 172:888-93.

47. Kowalski M, Jones N, Jewett MAS, et al. Treatment with intravesical Vicinium. ${ }^{T M}$ Results in Durable Responses in Patients with Carcinoma in situ (CIS) Previously Treated With BCG. Paper presented at the 30th Congress of the Société Internationale d'Urologie; November 1-5, 2009; Shanghai, China.
48. Huguet J, Crego M, Sabate S, et al. Cystectomy in patients with high risk superficial bladder tumours who fail intravesical BCG therapy: pre-cystectomy prostate involvement as a prognostic factor. Eur Urol 2005; $48: 53-9$.

49. Raj GV, Herr H, Serio AM, et al. Treatment paradigm shift may improve survival of patients with high risk superficial bladder cancer. J Urol 2007;177:1283-6.

50. Fahmy NM, Mahmud S, Aprikian AG. Delay in the surgical treatment of bladder cancer and survival: systematic review of the literature. Eur Urol 2006;50:1176-82.

Correspondence: Dr. Alexandre R. Zlotta, Department of Surgery, Division of Urology, University of Toronto, Mount Sinai Hospital and University Health Network, 60 Murray Street, 6th Floor, Box 19, Toronto, ON M5T 3L9; fax: 416-586-4776; azlotta@mtsinai.on.ca 